\title{
Electronic Two-Dimensional Beam Steering for Integrated Optical Phased Arrays
}

\author{
Behrooz Abiri, Firooz Aflatouni, Angad Rekhi, Ali Hajimiri \\ California Institute of Technology, 1200 E. California Blvd, Pasadena, CA 91125 \\ babiri@caltech.edu
}

\begin{abstract}
This paper presents electrical beam steering in an integrated 4x4 2D optical phased array (OPA) on a silicon on insulator (SOI) process enabling fast and repeatable beam steering for next generation projection, tracking, and imaging.

OCIS codes: (130.0130) Integrated optics; (130.6750) Systems; (110.5100) Phased-array imaging systems; (060.26051))

Free-space optical communication; (130.4815) Optical switching devices;
\end{abstract}

\section{Introduction}

Electronically steered phased array transmitters and receivers in microwave and mm-wave range have been widely used in radar systems where their agile electronic control enables rapid tracking of multiple fast moving objects [1]. Recently high frequency miniaturized phased array transmitters and receivers have been subject of interest for ranging, imaging, and directional communication at microwave, mm-wave, and $\mathrm{THz}$ frequencies [2-4]. Optical phased arrays (OPA) based on the same principle at much smaller wavelength have promising prospects for ranging, communications, image projection, optical network switch boxes, free-space optical computation, etc. Since optical wavelengths are much smaller than their RF counterparts, larger number of elements can be integrated on a smaller chip. Recent developments have shown the feasibility of current silicon-based micro-fabrication for integrated OPA implementation [5-8]. Beam steering can be achieved using thermo-optic phase shifters where the delay for each path is changed via the thermal index change [6,7]. In this approach, the large thermal time constants limit the speed at which the beam can be steered making them unsuitable for real-time and fast applications, such as projection, optical switches, or computing. Thermal crosstalk between adjacent phase shifters presents another challenge to the thermal phase control approach, necessitating remedial action, such as large separation between thermal phase shifters that can limit the number of elements in the OPA. In [6], the second dimension of beam steering is achieved via an external change of the wavelength. MEMS based OPAs [8] overcome the cross talk issue; however, they still have limited operation speed and lower reliability due to limitation of mechanical structures.

To overcome the speed and reliability limitations, PIN diode phase shifters that operate based on free carrier injection [9] are used in a 4x4 OPA in this work. Also, some level of amplitude control can be achieved, which provides side-lobe cancellation in the array operation. The utilized PIN-diode-based modulators are capable of operation with time constants under 1 nanosecond, thereby enabling very rapid response.

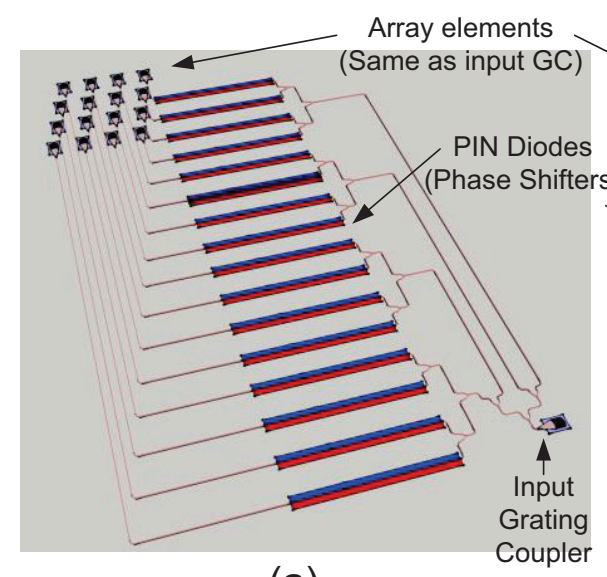

(a)

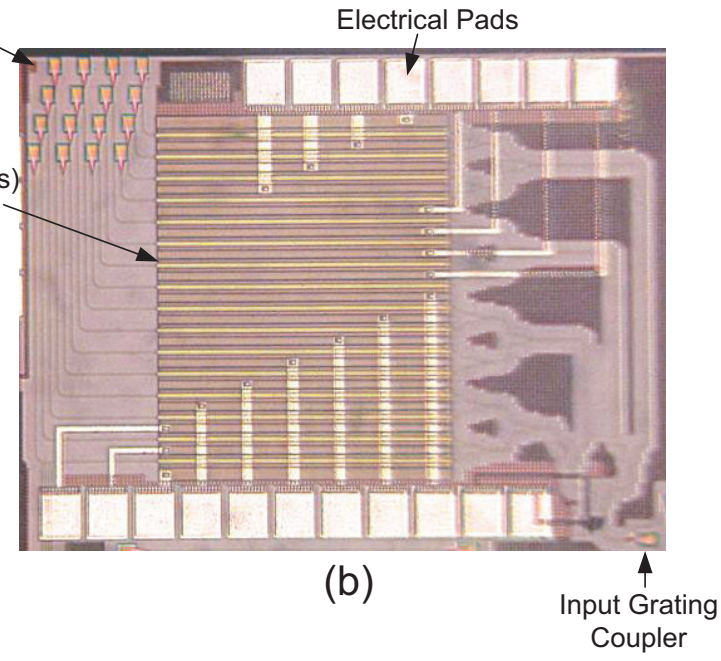

(b)

Fig. 1. (a) Layout of $4 \times 4$ OPA and (b) the die photo of the fabricated chip

\section{Design and Fabrication}

Fig. 1(a) shows the layout of the $4 \times 4$ OPA. The input light passes through a chain of $Y$-junctions that split the light into 16 waveguides. The light in each waveguide then passes through 16 PIN diodes that provide the phase control for each of the array elements. Each PIN diode is a $500 \mu \mathrm{m}$ long rib waveguide with doped slab and undoped strip, 
resulting in a phase shifter with $\mathrm{I}_{\pi} \approx 10.2 \mathrm{~mA}$. Sixteen grating couplers are used as radiating elements of the OPA. The peak radiation power of the grating couplers is achieved at 17 degrees at wavelength of $1550 \mathrm{~nm}$ with respect to normal. The horizontal and vertical spacing of the elements are $50 \mu \mathrm{m}$, however, the elements of each row are placed at an offset of $12.5 \mu \mathrm{m}$ with respect to the upper row to accommodate the optical routing.

The chip is fabricated in a SOI technology with minimum feature size of $100 \mathrm{~nm}$ and with $220 \mathrm{~nm}$ of silicon layer and $2 \mu \mathrm{m}$ buried oxide. The total area of the chip is $1.1 \times 0.9 \mathrm{~mm}^{2}$.

\section{Experimental Setup and Measurement Results}

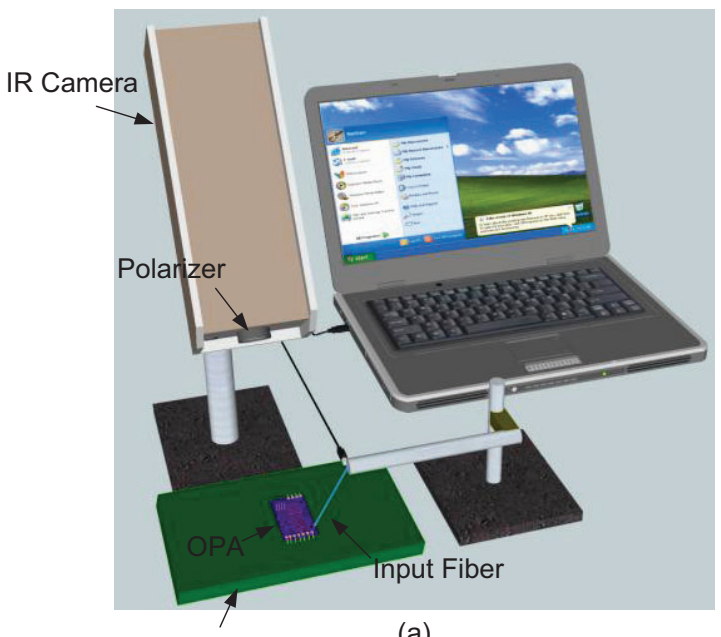

Control Board

(a)

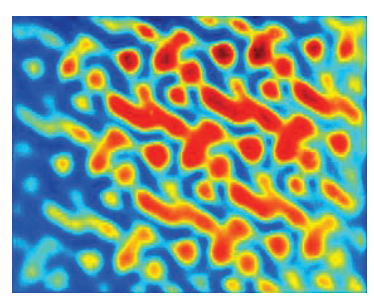

(b)

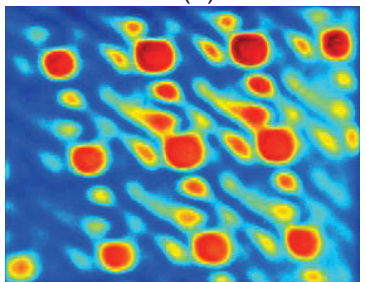

(c)

Fig. 2. (a) Experimental setup. (b) Initial random phased array pattern due to process variation. (c) Beam forming after adjusting phase shifters Fig. 2(a) shows the experiment setup. The OPA is mounted on a control board that converts the digital data from the computer to the electrical current for the phase shifters. A C-band DFB laser is used as the input light source and an IR camera is mounted around $10 \mathrm{~cm}$ above the chip to record the far field pattern from the OPA. A polarizer is placed between the chip and camera in order to block interference caused by reflection from input grating coupler to the camera.
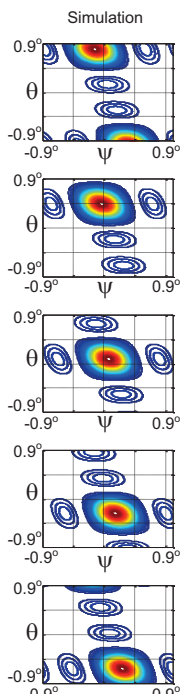

(a)

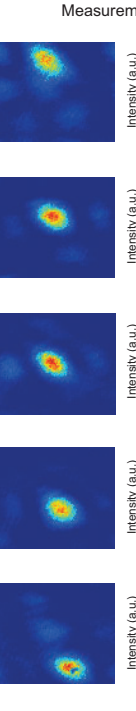

(b)
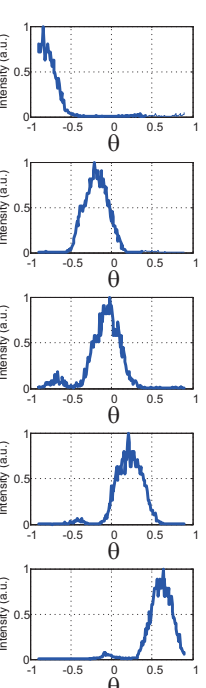

(c)

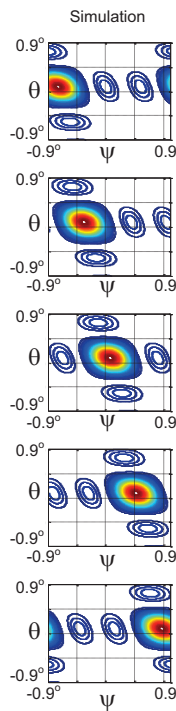

(d)



(e)

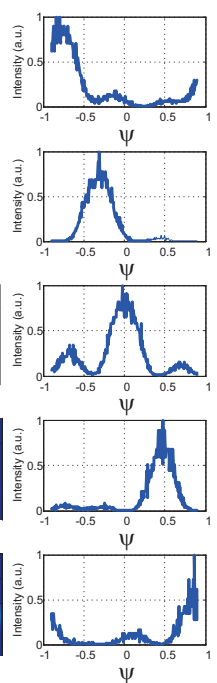

(f)
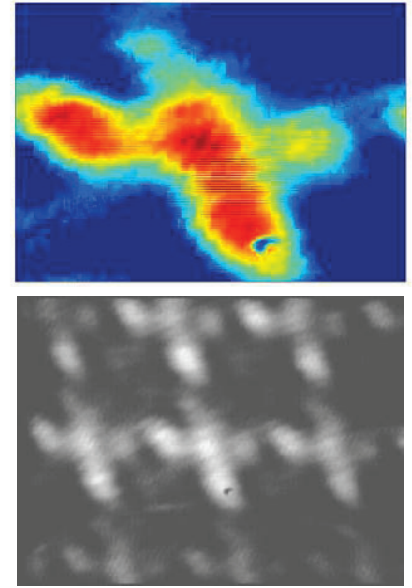

(g)

Fig. 3. Demonstration of real-time beam steering in OPA at $1553 \mathrm{~nm}$. (a) Simulation of vertical beam steering. (b), (c) Measured optimized pattern of vertical beam steering. (d) Simulation of horizontal beam steering. (e), (f) Measured optimized pattern of horizontal beam steering. (g) Fast beam steering of all the spots captured in a single frame of the camera to form a "+" sign.

All the paths to the OPA elements of the same row are designed and laid out to have equal length from the input to the output couplers. Also, the path length difference of adjacent rows is kept the same. Thus, the fabricated OPA would have a well-defined beam pattern without applying any current to the phase shifters, if there were no 
fabrication errors. However, mismatches in fabrication of optical waveguides and phase shifters results in optical phase mismatches between each radiating element. As a result, each fabricated chip shows a different far field pattern when the phase shifters are not activated. Fig. 2(b) shows an example of a random pattern that is observed from one of the unadjusted OPA chips. Due the large number of possible settings for phase shifters, a brute force approach cannot be used to find the correct biasing current for the phase shifters. We use a gradient descent optimization algorithm that compares the measured pattern from the camera with a desired simulated pattern and finds the phase shifter current setting that produces a similar pattern. Fig. 2(c) shows the pattern of the same OPA after phase adjustment.

In order to steer the optical beam, the optimizer is used to generate a lookup table for each steering angle as shown in Fig. 3(a-f). The values of the lookup table are then used to raster scan a "+" sign. Fig. 3(g) shows the resulting plus sign captured in a single frame of the camera with $20 \mathrm{~ms}$ of exposure time.

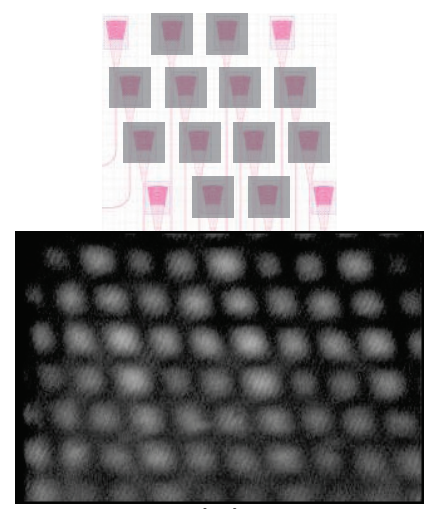

(a)
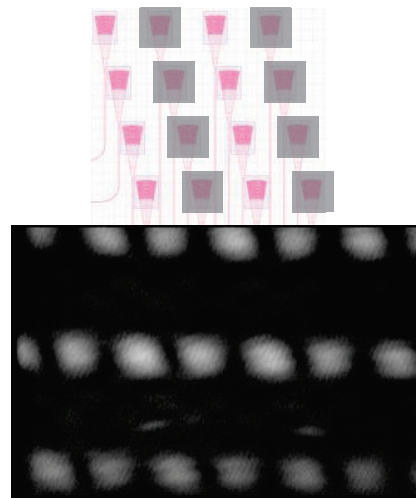

(b)

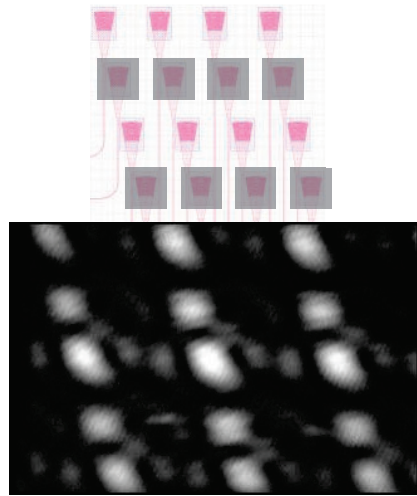

(c)

Fig. 4. Controlling amplitudes of OPA elements. (a) All elements except the four corner elements turned off. (b) Every other column turned off. (c) Every other row turned off.

The PIN phase shifter introduces around $0.25 \mathrm{~dB} / \mathrm{mA}$ of optical attenuation. This can be taken advantage to control the amplitudes of array elements and create more patterns. A few of possible patterns that can be generated by turning off some of the elements are shown in Fig. 4. Fig. 4(a) shows the pattern of a four element OPA created by attenuating all the elements except for the ones on the corners of the original OPA. Fig. 4(b) and (c) show the beam forming resulting from attenuating every other column and row, respectively.

\section{Summary and Conclusion}

In this paper we have demonstrated the first integrated OPA on silicon with fast beam steering capabilities to project images. The beam can be steered the full range (no blind spots). Pattern generation using rapid raster scanning has been demonstrated. An optimization method was used to calibrate phase of each element and compensate for fabrication mismatches in the phase shifters and waveguides.

\section{Acknowledgement}

The authors would like to thank OpSIS for chip fabrication.

\section{References}

[1] E. Taenzer, "Tracking multiple targets simultaneously with a phased array radar." Aerospace and Electronic Systems, IEEE Transactions on 5 (1980): 604-614.

[2] A. Natarajan, A. Komijani, and A. Hajimiri. "A fully integrated 24-GHz phased-array transmitter in CMOS." Solid-State Circuits, IEEE Journal of 40, no. 12 (2005): 2502-2514.

[3] C. H. Tseng, C. J. Chen, and T. H. Chu. "A low-cost 60-GHz switched-beam patch antenna array with Butler matrix network." Antennas and Wireless Propagation Letters, IEEE 7 (2008): 432-435

[4] K. Sengupta, and A. Hajimiri. "A 0.28 THz Power-Generation and Beam-Steering Array in CMOS Based on Distributed Active Radiators." (2012)

[5] K. Van Acoleyen, H. Rogier, and R. Baets. "Two-dimensional optical phased array antenna on silicon-on-insulator." Optics Express 18, no. 13 (2010): 13655-13660.

[6] J. Doylend, M. R. Heck, J. Bovington, J. Peters, L. Coldren, and J. Bowers. "Free-space Beam Steering in Two Dimensions Using a Silicon Optical Phased Array." In Optical Fiber Communication Conference. Optical Society of America, 2012.

[7] J. Sun, E. Timurdogan, A. Yaacobi, E. Shah Hosseini, and M. R. Watts. "Large-scale nanophotonic phased array." Nature 493, no. 7431 (2013): 195-199.

[8] B. W. Yoo, M. Megens, T. Chan, T. Sun, W. Yang, C. J. Chang-Hasnain, D. A. Horsley, and M. C. Wu. "Optical phased array using high contrast gratings for two dimensional beamforming and beamsteering." Optics express 21, no. 10 (2013): 12238-12248.

[9] G. T. Reed, G. Mashanovich, F. Y. Gardes, and D. J. Thomson. "Silicon optical modulators." Nature Photonics 4, no. 8 (2010): 518 -526. 